\title{
Malignant Transformation of Clival Chordoma After Gamma Knife Surgery
}

\author{
-Case Report-
}

\author{
Yoshifumi TsUBOI, Nakamasa HAYASHI, Masanori KURIMOTO, Shoichi NAGAI, \\ Masakiyo SASAHARA*, and Shunro ENDO \\ Departments of Neurosurgery and *Pathology, Faculty of Medicine, \\ University of Toyama, Toyama
}

\begin{abstract}
A 54-year-old woman presented a midline clival tumor manifesting as right abducens palsy in May 1997. Magnetic resonance (MR) imaging revealed a midline clival tumor. She underwent surgery twice with the transsphenoidal approach and gamma knife surgery for residual tumor. The histological diagnosis was chordoma. MR imaging revealed that the tumor had extended to the right cerebellopontine angle, with spinal seeding in February 2002. She underwent partial removal of the right cerebellopontine angle tumor. The histological diagnosis was chordoma with slight nuclear atypism. She died 5 years and 5 months after the first gamma knife surgery. Autopsy revealed multiple areas of spinal seeding. Histological examination confirmed malignant transformation with unique epithelial characteristics, possibly caused by gamma knife surgery.
\end{abstract}

Key words: clival chordoma, gamma knife surgery, malignant transformation, spinal seeding

\section{Introduction}

Chordomas are relatively rare, constituting only $0.1 \%$ to $0.2 \%$ of all intracranial tumors, ${ }^{16)}$ originate from remnants of the embryonic notochord, and are usually localized to the midline body. Approximately $50 \%$ of chordomas arise in the sacrococcygeal area, $35 \%$ in the spheno-occipital region, and the remaining $15 \%$ in other parts of the vertebral column ${ }^{5}{ }^{5}$ Chordomas are generally considered locally aggressive, with a high incidence of recurrence and relatively low rate of metastasis ranging from $3 \%$ to $43 \% .^{3,5,19,23,25)}$ The average time to metastasis is 3 years from the time when the primary tumor is identified. ${ }^{9)}$ Lungs and lymph nodes are the most common sites of metastasis, followed closely by the liver and bone. However, spinal seeding from a skull base chordoma is very rare. Clival chordomas are difficult to treat, because of the high rate of recurrence and contiguity with vital structures, such as the brainstem, basilar artery, pituitary gland, cavernous sinus, and cranial nerves. Malignant transformation in chordomas has an incidence of only $4 \%,{ }^{20)}$

Received November 30, 2006; Accepted July 18, 2007 and generally manifests as sarcomatous changes.

We report a case of spinal seeding from a clival chordoma, with malignant transformation with epithelial characteristics following gamma knife surgery.

\section{Case Presentation}

A 54-year-old woman presented with double vision in May 1997. Her past history and family history were uneventful. Neurological examination revealed 6th cranial nerve palsy. Computed tomography with contrast medium revealed a tumor in the clivus. Magnetic resonance (MR) imaging revealed a midline clival tumor with bone destruction, appearing as hypointense on $\mathrm{T}_{1}$-weighted and hyperintense on $\mathrm{T}_{2}$-weighted images. $\mathrm{T}_{1}$-weighted MR imaging with gadolinium revealed homogeneous enhancement of the lesion (Fig. 1A). She underwent surgery with a transsphenoidal approach under general anesthesia in November 1997. The histological diagnosis was chordoma (Fig. 2A). Postoperative MR imaging revealed residual tumor, so the patient underwent gamma knife surgery (peripheral dose $17 \mathrm{~Gy}$, maximum core dose $34 \mathrm{~Gy}$ ) in December 1997. MR imaging revealed regrowth 

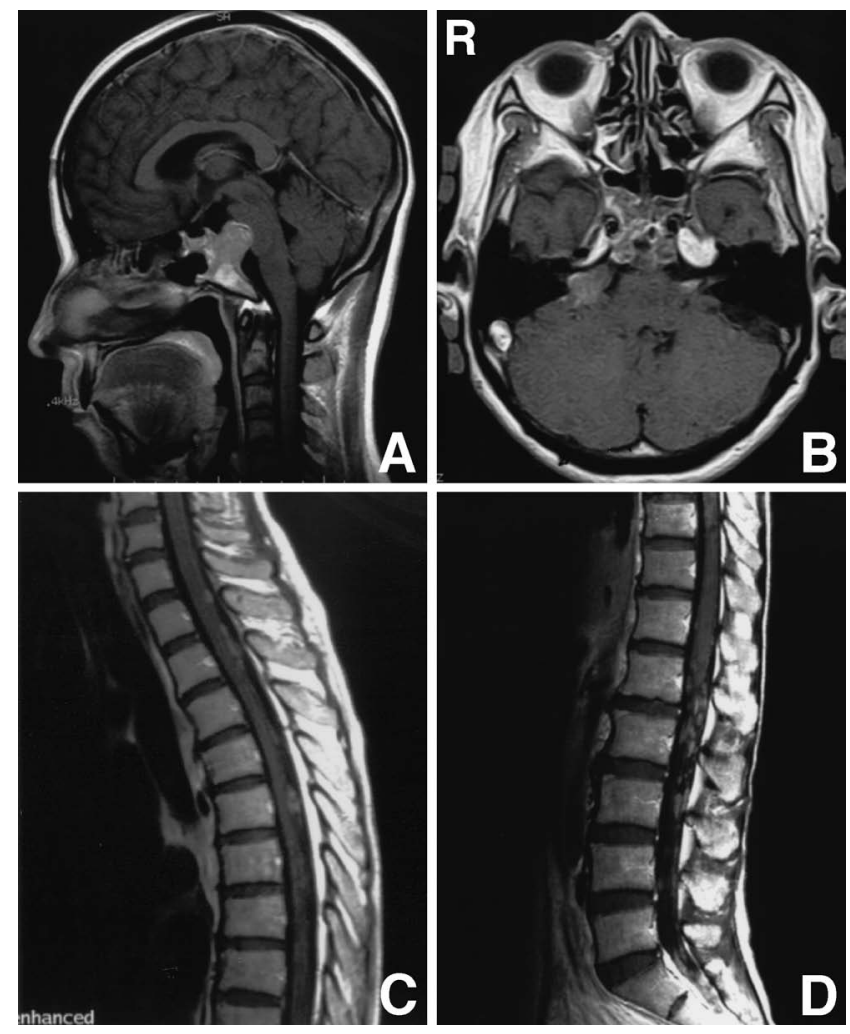

Fig. 1 T $\mathrm{T}_{1}$-weighted magnetic resonance images with contrast medium at first admission showing a midline clival tumor (A), and extension of the tumor into right cerebellopontine angle (B) and spinal seeding in the thoracic (C) and lumbar cord (D) at 4 years after the first operation.

of the residual tumor in February 2001. She underwent second gamma knife surgery (peripheral dose 16 Gy, maximum core dose 32 Gy) in February 2001. She complained of right facial palsy and dysphagia in February 2002. MR imaging revealed extension of the tumor to the right cerebellopontine angle and spinal seeding in the cervical, thoracic, and lumbar cord (Fig. 1B-D). Although she underwent surgery for the right cerebellopontine angle tumor, only partial resection was possible. The histological diagnosis was chordoma with slight nuclear atypism. She died 5 years and 5 months after the first gamma knife surgery. At autopsy, clival tumor was found extending to the right cerebellopontine angle, and compressing the brainstem and cerebellum. Multiple areas of spinal seeding were found in the cervical, thoracic, and lumbar cord (Fig. 3). No findings of metastases were detected in other organs.

The primary tumor consisted of sheets of physaliphorous cells. The histological diagnosis was chordoma (Fig. 2A). No mitosis or necrosis was ob-

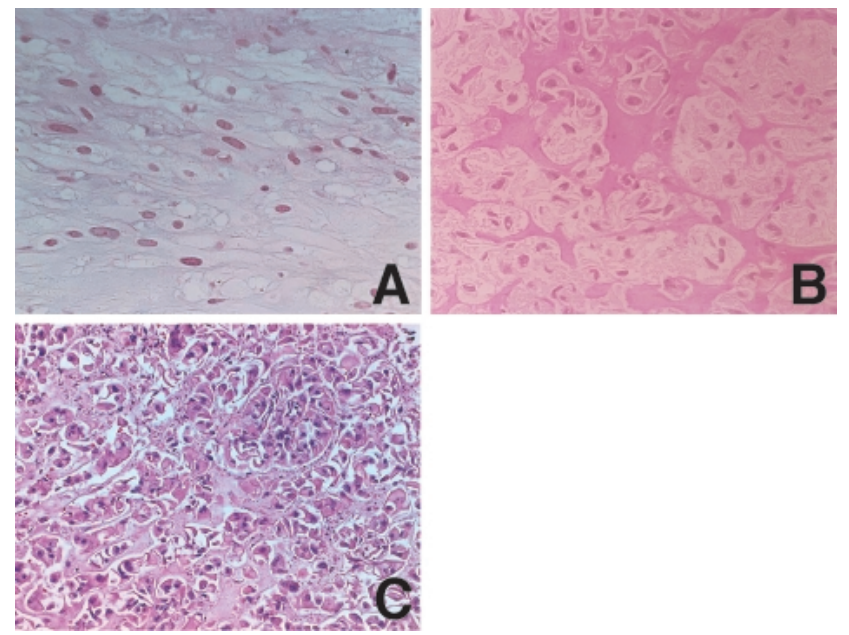

Fig. 2 Photomicrographs showing the tumor cells contained physaliphorous cells and nuclear atypism at the first operation (A: hematoxylin and eosin [HE] stain, $\times 400$ ), physaliphorous cells in mucous matrix with slightly nuclear atypism at the second operation (B: HE stain, $\times 400$ ), and atypical nuclei and prominent nucleoli at autopsy (C: HE stain, $\times 200$ ).

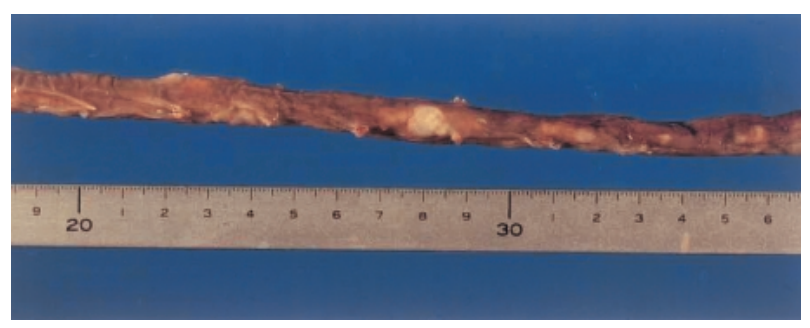

Fig. 3 Photograph showing nodules on multiple areas of the spinal cord.

served. The MIB-1 staining index was $2.4 \%$. The tumor cells resected at the second operation had eosinophilic cytoplasm (Fig. 2B). Physaliphorous cells were found in the mucous matrix. Slight nuclear atypism but no mitosis or necrosis was observed. The MIB-1 staining index was $4.4 \%$.

Autopsy found that most of the tumor lesion irradiated by gamma knife surgery consisted of necrotic tissue, as well as sheet-like architecture with nuclear pleomorphism. The metastatic lesion of the spinal cord also exhibited sheet-like architecture and a nest-forming pattern. The tumor cells contained atypical nuclei and prominent nucleoli (Fig. 2C). Necrosis and mitoses were observed. These findings indicated malignant transformation of epithelial nature. Immunohistochemical examina- 
Table 1 Previous cases of spinal seeding from skull base chordoma

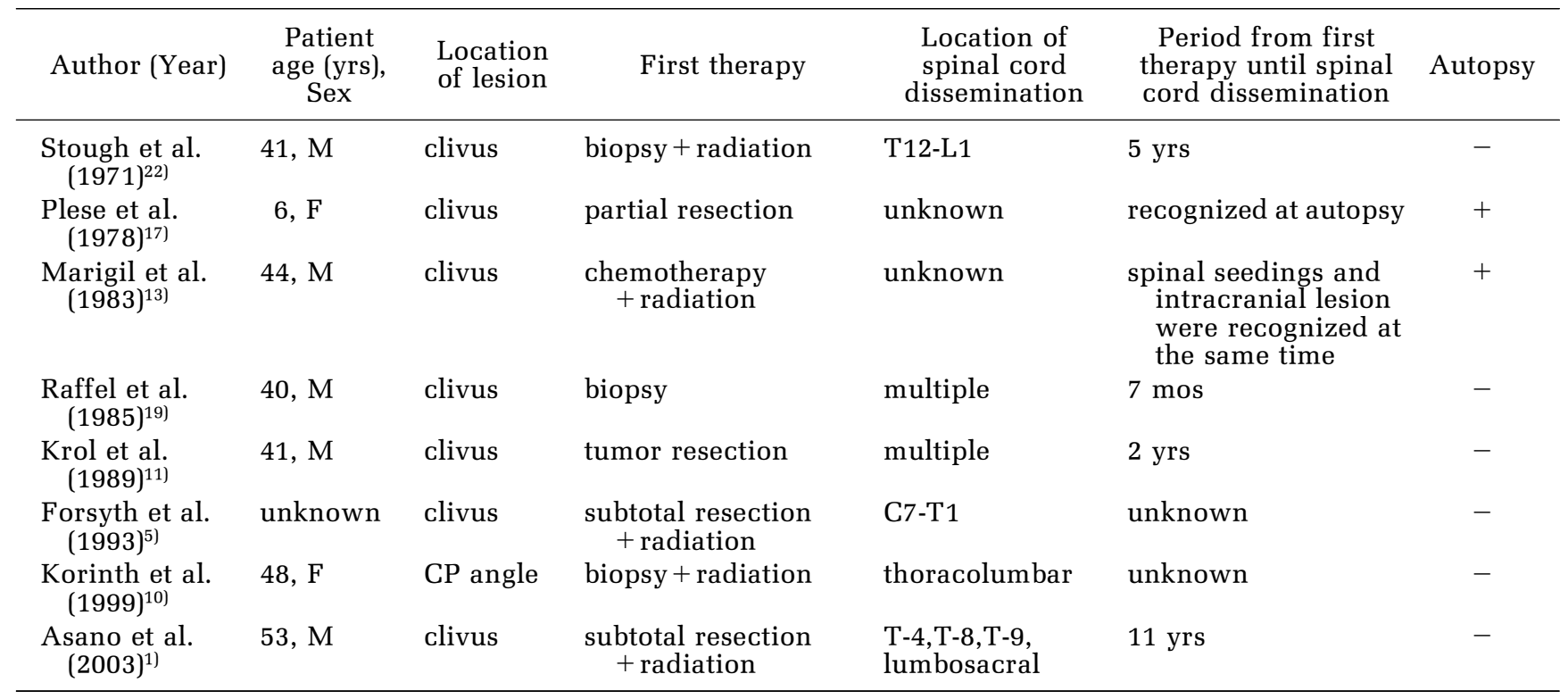

CP: cerebellopontine.

tion showed the tumor was positive for epithelial membrane antigen (EMA) and cytokeratin, and faintly positive for vimentin and S-100 protein, supporting the epithelial nature. The MIB-1 staining index was $11 \%$.

\section{Discussion}

Only eight cases of spinal seeding from a skull base chordoma have been reported (Table 1).1,5,10,11,13,17, 19,22) Spinal seeding was identified at autopsy in two cases, simultaneously with the clival lesion in one case, and during observation ranging from 7 months to 11 years in six cases. Initial treatment was surgical resection in three cases, surgery and radiation in four cases, and chemotherapy and radiation in one case. The histological findings were progressive features including pleomorphism, malignant cell, and mitosis in the three surgical cases. Two factors may be related to spinal seeding: destruction of the dura mater during the surgical procedure and malignant transformation of the tumor. Destruction of the dura mater may result in dissemination of the tumor in the skull base to the spinal cord. In the present case, there were no findings of tearing of the dura mater or leakage of cerebrospinal fluid during the surgery. However, microdestruction of the dura mater may have occurred. Chordoma arises from cartilage remnants of the primordial skull base and initially grows extradurally, but can penetrate the dura. ${ }^{20)}$ In addition, chordoma tends to invade the dura and lep- tomeninges. ${ }^{13)}$ Malignant transformation may accelerate such character and result in spinal seeding, as more anaplastic chordomas are more likely to metastasize. ${ }^{3)}$

Malignant transformation of chordoma is quite rare. Only two of 52 cases of cranial chordoma exhibited malignant transformation, after irradiation in one case, and de novo transformation to sarcoma in the other. ${ }^{23)}$ There have been some reports of malignant transformation occurring after administration of radiation ${ }^{2,6,8,15,23)}$ and de novo malignant transformation. ${ }^{14)}$ Malignant transformation is usually associated with sarcomatous change. ${ }^{23)}$ The histological findings of malignant transformation after radiation and de novo malignant transformation show no obvious differences., ${ }^{2,6,8,14,15,23)}$ Malignant progression of benign brain tumors may occur after gamma knife radiosurgery, but the primary neoplasm should be considered as a biologically aggressive tumor rather than the side effects of gamma knife radiosurgery. ${ }^{12)}$ In the present case, the MIB-1 staining index was $2.4 \%$ in the first specimen, and increased up to $11 \%$ in the autopsy specimen.

The typical immunohistochemical staining pattern of sarcoma is negative for EMA and cytokeratin, and positive for S-100 protein and vimentin. ${ }^{18,23)}$ However, the present tumor was positive for EMA and cytokeratin, and faintly positive for vimentin and S-100 protein, which demonstrated the epithelial nature. Several theories have been proposed to explain sarcomatous change, including arising adjacent to irradiated chordoma, arising from the 
mesenchymal component of a chordoma, arising from mesenchyme unrelated to the chordoma, and arising from the epithelial component of a chordoma. ${ }^{8)}$ In this case, malignant transformation with epithelial nature may have occurred by a different pathogenesis.

The risk of radiation-induced tumors after radiotherapy for benign disease is $0.5-3 \%$ after 30 years. ${ }^{4)}$ Malignant transformation of acoustic neurinoma and meningeal melanocytoma after gamma knife surgery have been reported,7,21,24) but not malignant transformation of chordoma. The malignant transformation in the present case exhibited unique characteristics, and could have been caused by the gamma knife surgery. The possibility of spinal seeding and malignant transformation should be considered after gamma knife surgery for skull base chordoma.

\section{References}

1) Asano S, Kawahara N, Kirino $T$ : Intradural spinal seeding of a clival chordoma. Acta Neurochir (Wien) 145: 599-603, 2003

2) Belza MG, Urich $\mathrm{H}$ : Chordoma and malignant fibrous histiocytoma: Evidence for transformation. Cancer 58: 1082-1087, 1986

3) Chambers PW, Schwinn CP: A clinicopathologic study of metastasis. Am J Clin Pathol 72: 765-776, 1979

4) Evans DGR, Birch JM, Romsden RT, Sharif S, Baser ME: Malignant transformation and new primary tumours after therapeutic radiation for benign disease: substantial risks in certain tumour prone syndromes. J Med Genet 43: 289-294, 2006

5) Forsyth PA, Cascino TL, Shaw EG, Scheithauer BW, O'Fallon JR, Dozier JC, Piepgras DG: Intracranial chordomas: a clinicopathological and prognostic study of 51 cases. J Neurosurg 78: 741-747, 1993

6) Halpern J, Kopolovic J, Catane R: Malignant fibrous histiocytoma developing in irradiated sacral chordoma. Cancer 53: 2661-2662, 1984

7) Hanabusa K, Morikawa A, Murata T, Taki W: Acoustic neurinoma with malignant transformation. Case report. J Neurosurg 95: 518-521, 2001

8) Hruban RH, May M, Marcove RC, Huvos AG: Lumbo-sacral chordoma with high-grade malignant cartilaginous and spindle cell components. Am J Surg Pathol 14: 384-389, 1990

9) Huvos AG: Bone Tumors, Diagnosis, Treatment and Prognosis, ed 2. Philadelphia, Saunders, 1991, pp 599-624

10) Korinth M, Schonrock L, Mayfrank L, Gilsbach JM: Primary intradural pontocerebellar chordoma metastasizing in the subarachnoid spinal canal. Zentralbl Neurochir 60: 146-150, 1999

11) Krol G, Sze G, Arbit E, Marcove R, Sundaresan N: Intradural metastases of chordoma. AJNR Am J Neu- roradiol 10: 193-195, 1989

12) Kubo O, Chernov M, Izawa M, Hayashi M, Muragaki Y, Maruyama T, Hori T, Takakura K: Malignant progression of benign brain tumors after gamma knife radiosurgery: Is it really caused by irradiation? Minim Invasive Neurosurg 48: 334-339, 2005

13) Marigil MA, Pardo-Mindan FJ, Joly M: Diagnosis of chordoma by cytologic examination of cerebrospinal fluid. Am J Clin Pathol 80: 402-404, 1983

14) Meis JM, Raymond AK, Evans HL, Charles RE, Giraldo AA: Dedifferentiated chordoma: a clinicopathologic and immunohistochemical study of three cases. Am J Surg Pathol 11: 516-525, 1987

15) Miettinen M, Lehto VP, Virtanen I: Malignant fibrous histiocytoma within a recurrent chordoma. A light microscopic, electron microscopic, and immunohistochemical study. Am J Clin Pathol 82: 738-743, 1984

16) Pallini R, Maira G, Pierconti F, Falchetti ML, Alvino E, Cimino-Reale G, Fernandez E, D'Ambrosio E, Larocca LM: Chordoma of the skull base: predictors of tumor recurrence. J Neurosurg 98: 812-822, 2003

17) Plese JPP, Borges JM, Nudelman M, Lefevre AB, Sallum J: Unusual subarachnoid metastasis of an intracranial chordoma in infancy. Childs Brain 4: 251-256, 1978

18) Radner H, Katenkamp D, Reifenberger G, Deckert M, Pietsch T, Wiestler OD: New developments in the pathology of skull base tumors. Virchows Arch 438: 321-335, 2001

19) Raffel C, Wright DC, Gutin PH, Wilson CB: Cranial chordomas: clinical presentation and results of operative and radiation therapy in twenty-six patients. Neurosurgery 17: 703-710, 1985

20) Samii M, Draf W: Surgery of the Skull Base. Berlin, Heidelberg, Springer-Verlag, 1989, pp 90-101

21) Shin M, Ueki K, Kurita H, Kirino T: Malignant transformation of a vestibular schwannoma after gamma knife radiosurgery. Lancet 360: 309-310, 2002

22) Stough DR, Hartzog JT, Fisher RG: Unusual intradural spinal metastasis of a cranial chordoma. J Neurosurg 34: 560-562, 1971

23) Tomlinson FH, Scheithauer BW, Forsythe PA, Unni KK, Meyer FB: Sarcomatous transformation in cranial chordoma. Neurosurgery 31: 13-18, 1982

24) Uozumi Y, Kawano $T$, Kawaguchi $T$ Ooasa $T$, Ogasawara S, Yoshida H, Yoshida T: Malignant transformation of meningeal melanocytoma: a case report. Brain Tumor Pathol 20: 21-25, 2004

25) Volpe R, Mazabraud A: A clinicopathologic review of 25 cases of chordoma (a pleomorphic and metastasizing neoplasm). Am J Surg Pathol 7: 161-170, 1983

Address reprint requests to: Nakamasa Hayashi, M.D., Department of Neurosurgery, Faculty of Medicine, University of Toyama, 2630 Sugitani, Toyama 930-0194, Japan.

e-mail:nakamasa@ms.toyama-mpu.ac.jp 